\title{
Comparison of various diagnostic methods in assessing platelet count in patients with immune thrombocytopenia
}

\author{
Michał Witkowski ${ }^{1 *}$, Magdalena Witkowska ${ }^{2}$, Marzena Tybura-Sawicka ${ }^{3}$, Agata Majchrzak ${ }^{1}$, \\ Tadeusz Robak ${ }^{4}$, Piotr Smolewski ${ }^{2}$
}

${ }^{1}$ Department of Hematology, Copernicus Memorial Hospital, Lodz, Poland

${ }^{2}$ Department of Experimental Hematology, Medical University of Lodz, Poland

${ }^{3}$ Department of Hemostasis Disorders, Medical University of Lodz, Poland

${ }^{4}$ Hematology Clinic, Medical University of Lodz, Poland

\begin{abstract}
Introduction: Accurate platelet count (PLTC) in immune thrombocytopenia (ITP) is important in order to make therapeutic decisions. The basic method of assessing PLTC is peripheral blood morphology with EDTA or with citrate. The older way of assessing PLTC is measurement under the microscope (FONIO), and the newer way is the fluorescent method. The purpose of this study was to compare PLTC methods, and find the most reliable.

Material and methods: PLTC was assessed using five methods (EDTA, citrate, FONIO, fluorescent, and immunofluorescent methods) in adult patients with previously untreated ITP.

Results: 66 patients were enrolled in the study. The median age was 56 years and $56 \%$ were men. Median PLTC in EDTA was $69 \mathrm{G} / \mathrm{L}$, in citrate $69 \mathrm{G} / \mathrm{L}$, in fluorescence $69 \mathrm{G} / \mathrm{L}$, in FONIO $90 \mathrm{G} / \mathrm{L}$, and in immunofluorescence $83 \mathrm{G} / \mathrm{L}$. A significant difference in PLTC was observed in comparing EDTA to immunofluorescence $(53 \% \pm 123 \%)$, followed by FONIO (51\% $\pm 91 \%$ ), PLTC from immunofluorescence differed from the fluorescent method by $40 \% \pm 78 \%$.

Conclusions: The most valuable method for obtaining PLTC is the immunofluorescent method. These findings are especially important in helping to make therapeutic decisions during a challenging time for accessing medical care such as a pandemic.
\end{abstract}

Key words: flow cytometry, immunofluorescence, immune thrombocytopenia, platelet count

Acta Haematologica Polonica 2021; 52, 6: 584-589

\section{Introduction}

Immune thrombocytopenia (ITP) is an acquired disease characterized by isolated low platelet count (PLTC) and an increased risk of bleeding [1]. The pathophysiology underlying this disease is still not completely understood. So far, several disease mechanisms have been proposed, but the most plausible includes increased platelet destruction due to sensitization of platelets by autoantibodies. It has been observed that these autoantibodies are mainly lgG, usually connected with IgM, directed against glycoprotein (GP) complexes IIb/IIla, GPIb/IX and GPla/Ila [2].

\footnotetext{
*Address for correspondence: Michał Witkowski, Department of Hematology, Copernicus Memorial Hospital, Ciolkowskiego 2, 93-513 Lodz, Poland, phone +48 426895191 e-mail: michalwitkowski13@gmail.com
}

PTHiT Copyright $\odot 2021$

The Polish Society of Haematologists and Transfusiologists, Insitute of Haematology and Transfusion Medicine. All rights reserved.

Received: 22.02.2021 Accepted: 30.03.2021

This article is available in open access under Creative Common Attribution-Non-Commercial-No Derivatives 4.0 International (CC BY-NC-ND 4.0) license, allowing to download articles and share them with others as long as they credit the authors and the publisher, but without permission to change them in any way or use them commercially. 
ITP can be classified as 'primary' when occurring without any underlying disease, or 'secondary' when associated with another disease such as connective tissue disorder, a viral infection, or certain drugs. According to the American Society of Hematology (ASH) guidelines, ITP is diagnosed by identifying PLTC of less than $100 \mathrm{G} / \mathrm{L}$ in the absence of other causes or disorders [3].

Despite evidence that the PLTC by itself has limitations as an effective marker of the need for treatment, it is still the most commonly applied parameter in ITP patients. There is clinical value in differences in PLTC, especially when the amount is below $20-30 \mathrm{G} / \mathrm{L}$, because of the influence on therapeutic decisions like starting immunosuppressive treatment [4].

The basic method of assessing PLTC is peripheral blood morphology anticoagulated with ethylene diamine tetracetic acid (EDTA) or with citrate. The older version involves counting platelets under the microscope, while the newer is the fluorescent method. There is a need to identify better laboratory tests of bleeding risk, other than provided by PLTC, which fails to provide information on platelets aggregation and their function. The answer may be flow cytometry (FCM) with the immunofluorescent method, which until now has only been available in highly specialized centralized laboratories, but in future could be an inpatient testing method that might provide additional guidance [5].

To date, there has been no publication comparing all five different PLTC methods.

In our study, we evaluated and compared for the first time the five different methods of evaluating PLTC in ITP patients: peripheral blood morphology with EDTA and with citrate, counting platelets under the microscope, fluorescent and immunofluorescent methods. We believe this to be an important study, especially in the extremely challenging conditions of a pandemic.

\section{Material and methods}

Our study involved a group of 66 patients with an ITP diagnosis according to the ASH 2019 guidelines [3]. The inclusion criteria were: age $18+$, previously untreated, and primary disease. All the included patients had not received any medical treatment for at least one month before sampling. The group was composed of 27 women and 39 men, mean age 56 years [standard deviation (SD) 19]. In all patients, we evaluated PLTC using five different methods: EDTA and citrate in peripheral blood, manually under the microscope, and fluorescent and immunofluorescent methods.

About $5 \mathrm{~mL}$ of blood was drawn from a vein in the patient's inner elbow region. The freshly collected whole blood samples were divided into tubes with tripotassium salt of EDTA and trisodium citrate and counted for PLTC by an automatic hematology analyzer, Sysmex XN100 (Sysmex Corporation, Kobe, Japan). Precautions were taken to ensure there were no time lapses. After blood collection, it was analyzed within four hours. The calibration status of the Sysmex analyzer was initially checked by the manufacturer. Quality control samples and maintenance procedures were performed daily according to the manufacturer's instructions.

\section{Microscope method}

PLTC was also evaluated by manual platelet counting under the microscope (FONIO) [8]. A direct smear was made by placing a drop of blood onto a microscope slide and spreading it into a thin layer. Slides were stained with Giemsa stain, and reviewed for PLTC and platelet aggregates or clumping.

\section{Fluorescent method}

We also used the new fluorescent method (FFC) for counting platelets on the Sysmex XN1000 analyzer. In platelet counting by the FFC, platelets are stained with a fluorescent oxazine dye that is specifically bound with nucleic acid-rich platelet organelles, for example ribosomes or mitochondria. These are irradiated with a semiconductor laser beam, and then the forward scattered light and side fluorescence intensities of each platelet are plotted on a 2D scattergram to differentiate and count the platelets. FFC helps in specific differentiation of platelets from other blood cells and from interfering particles such as red blood cell fragments. Moreover, the analyzed sample volume of the FFC channel is about five times larger than those of standard methods. This provides highly precise data even in situations when the PLTC is extremely low.

\section{Immunofluorescent method}

Coagulation sodium citrate samples were used to evaluate the platelet count by the immunofluorescent method. The staining and gating protocol followed that proposed by the London Laboratory Service Group [7, 8] (Figure 1). Whole blood was incubated with FITC-labeled monoclonal antibody CD41a and CD61 (Becton Dickinson, San Jose, CA, USA). FCM measurements were carried out on FASC Lyric (Becton-Dickinson).

\section{Statistical evaluation}

For the statistical analysis of data obtained, the range of the measured variable, mean, median and SD were calculated, using statistical software (STATISTICA v.7.0, Tulsa, OK, USA). Data was presented as a median or mean SD values. The differences between values were evaluated with non-parametric Mann-Whitney test. For assessment of correlations, Spearman's rank correlation coefficient or Chi-squared test were performed where necessary. $P$ values of less than 0.05 were considered statistically significant. 


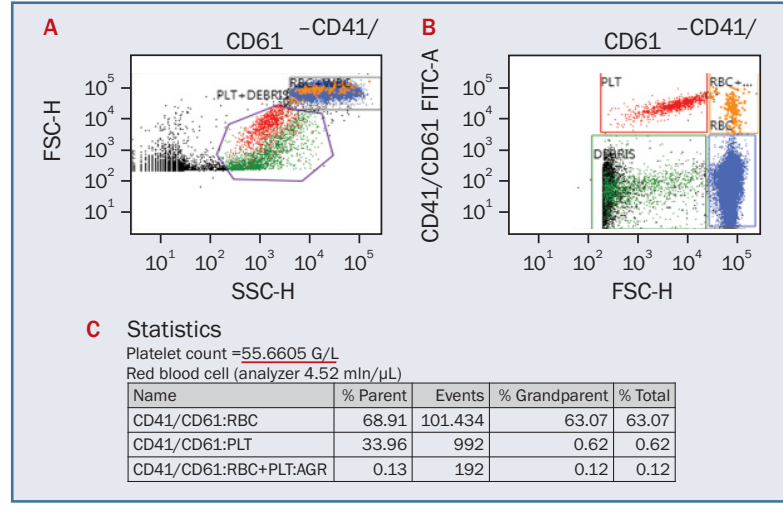

Figure 1. Gates used for analysis of platelets identified as CD61+ population (A, B). Statistical formula for platelet count in immunofluorescent method with flow cytometer (C); RBC - red blood cells; PLT - platelets; AGR - aggregates

\section{Ethics committee}

This study was approved by the local ethics committee, and all patients provided written informed consent to participate.

\section{Results}

We enrolled 66 patients with previously untreated ITP. The majority of patients were male $(n=37,56 \%)$, with median age at diagnosis of 56 years ( \pm 19 ). The median PLTC in the EDTA method was $69 \mathrm{G} / \mathrm{L}(1-164)$, with citrate $69 \mathrm{G} / \mathrm{L}$ (1-205), with fluorescence $69 \mathrm{G} / \mathrm{L}(2-164)$, with FONIO $90 \mathrm{G} / \mathrm{L}(1-250)$, and with immunofluorescence $83 \mathrm{G} / \mathrm{L}$ (5-283 SD \pm 65 ) (Figure 2). The characteristics of the patients are set out in Table I.

The standard method (PLTC in peripheral blood count with EDTA) was compared to the other available diagnostic methods including the FONIO, citrate morphology, fluorescent, and immunofluorescent methods. The mean and median of the measurements from the immunofluorescent method $(p=0.01)$ and the FONIO method $(p<0.01)$ were significantly higher than from the analyzer. The biggest difference in PLTC was observed in the results comparing the immunofluorescence $(53 \% \pm 123 \%)$, method followed by the FONIO method $(51 \% \pm 91 \%)$ (Figure 3). The mean and median of the measurements with FFC and citrate did not differ significantly from the measurement from the analyzer. No difference was observed in PLTC when comparing the standard method to the FFC $(7 \% \pm 43 \%)$ and the morphology with citrate $(7 \% \pm 58 \%)$. The PLTC results obtained with immunofluorescence differed from fluorescence by $40 \% \pm 78 \%$.

Because of the possibility of platelet aggregation, the obtained results were correlated with standard morphological platelet parameters [platelet distribution width (PDW), mean platelet volume (MPV), platelet large cell ratio

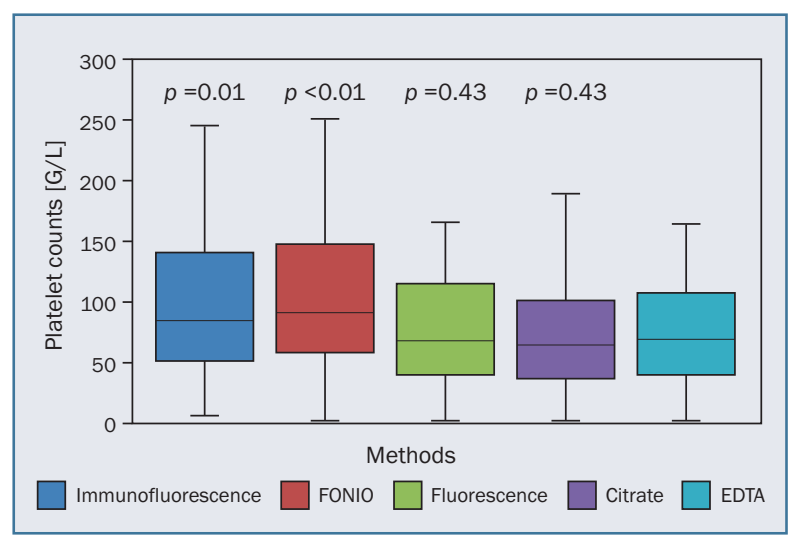

Figure 2. Platelet counts $[\mathrm{G} / \mathrm{L}]$ determined using immunofluorescence (dark blue), FONIO (red), fluorescence (green), citrate (violet), and standard analyzer ethylene diamine tetracetic acid (EDTA; light blue) methods

Table I. Characteristics of study group

\begin{tabular}{l|l}
\hline Parameter & Value \\
\hline $\begin{array}{l}\text { Number of patients } \\
\text { Age }\end{array}$ & $n=66$; male 56\% \\
Mean platelet level & $69(1-164) \mathrm{G} / \mathrm{L}$ \\
EDTA analyzer & $69(1-205) \mathrm{G} / \mathrm{L}$ \\
Citrate analyzer & $69(2-164) \mathrm{G} / \mathrm{L}$ \\
\hline Fluorescent method & $90(1-250) \mathrm{G} / \mathrm{L}$ \\
\hline Microscope (FONIO) & $83(5-283) \mathrm{G} / \mathrm{L}$ \\
\hline Immunofluorescent method & \\
\hline Platelet parameters & $16(9-25)$ \\
Platelet distribution width (PDW) [fL] & $12(9-15)$ \\
Mean platelet volume (MPV) [fL] & $41(10-59)$ \\
Platelet large cell ratio (P-LCR) [\%] & $18(3-42)$ \\
\hline Immature platelet fraction (IPF) [\%] & $5(3-6)$ \\
Red blood cell (RBC) [million/ $\mu \mathrm{L}]$ & $7(3-29)$ \\
White blood cell (WBC) [thousand/ $\mu \mathrm{L}]$ & $25.504(10.559-$ \\
Mean fluorescence intensity (MFI) & $-59.667)$ \\
\hline
\end{tabular}

EDTA - ethylene diamine tetracetic acid

(P-LCR)], with the number of white and red blood cells, with the fraction of immature platelets, and with the mean fluorescence intensity (MFI). There were no statistical differences observed in the measurements of the above-mentioned parameters.

\section{Discussion}

Determining the exact PLTC is crucial in ITP patients because of the decision to start treatment. To date, the most commonly used method to assess and observe PLTC in ITP diagnosis has been morphology for EDTA. This is a quick and 


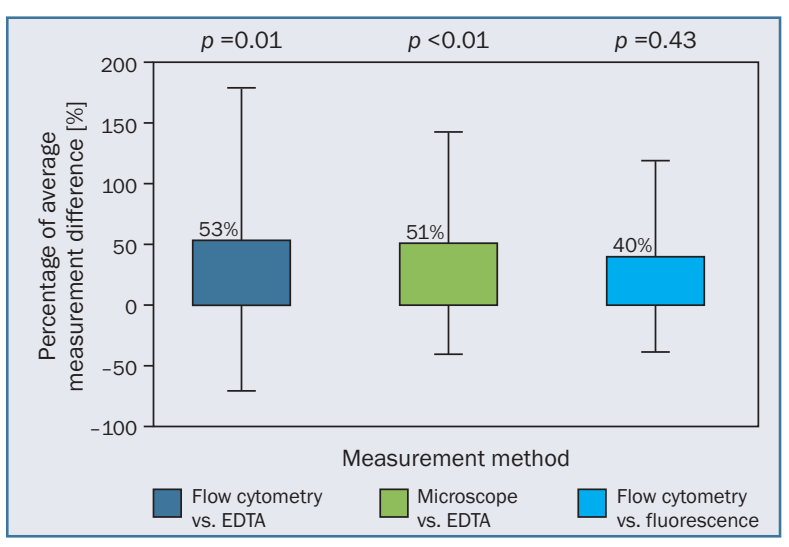

Figure 3. Comparison of mean differences in platelet count measurements depending on method; EDTA - ethylene diamine tetracetic acid

cheap method, but has unfortunately important drawbacks. One of them is EDTA-dependent pseudo-thrombocytopenia, a commonly known laboratory phenomenon. Its prevalence has been observed to vary between $0.1-2 \%$ among hospitalized patients and $15-17 \%$ in patients with an ITP diagnosis $[9,10]$. A possible reason for this phenomenon may be agglutinating antibodies that recognize cytoadhesive receptors on platelet GPIIb/IIla, which as a result cause platelet clumping [11].

We should be very careful in interpreting PLTC in ITP patients obtained with conventional EDTA methods which have limitations in platelet measurements such as poor accuracy and precision in the low PLTC and interference by nonplatelet particles.

The perfect method for ITP patients is still being sought. The best method of counting platelets in samples from ITP patients is still a matter of debate. Due to its high imprecision and laboriousness, the manual method has fallen out of favor and been replaced by immunological platelet counting using FCM. In the study by Gatt et al. [8], the PLTC was assessed by standard EDTA measurement and the immunofluorescent method. The results were similar to those observed in our study. The PLTC assessed by FCM was higher than that measured by the standard EDTA method (mean difference $4 \mathrm{G} / \mathrm{L}, p=-0.0011$ ). There was an excellent correlation between the counts determined by the EDTA and immunofluorescence $(r=0.89, p<0.0001)$. In the work by Bowles et al. [12] in ITP patients, measurement of the platelet count by the standard EDTA method frequently underestimated the PLTC as defined by the immunofluorescent method. In a group of 35 enrolled patients, the mean PLTC by standard EDTA method was $44 \mathrm{G} / \mathrm{L}$ and by immunofluorescence was $56 \mathrm{G} / \mathrm{L}(p<0.001)$. Similar results were observed by Harrison et al. [13].

Both these studies support our finding that the consistent common discrepancy in the EDTA method of evaluating ITP patients underestimates PLTC.
The platelet is an interesting but difficult cell to study. The immunofluorescent method provides a rapid, accurate and reproducible test for PLTC, and might be adopted by laboratories with appropriate FCM experience. One of the reasons why immunofluorescence may be more accurate and extremely useful in ITP patients is detecting the immature (reticulated) platelets $[14,15]$. These are not routinely assessed by hematology analyzers, but are crucial in the evaluation of the bone marrow response to thrombocytopenia.

A great hope for the future is the newly developed XN-Series automated hematology analyzer, equipped with a novel dedicated channel for platelet analysis, which is based on the FFC method. Tanaka et al. [16] observed that FFC gave a higher correlation with the immunofluorescent method compared to the standard EDTA method for samples with a PLTC $\leq 50 \mathrm{G} / \mathrm{L}$. The same results were noted by Sun et al. [17]. PLTC counted using FFC channel was much more accurate than other diagnostic methods including standard EDTA, especially in thrombocytopenic patients.

FFC may be a more precise and accurate method, even in ITP patients. Unfortunately, this observation was not confirmed in our study.

It has been observed that ITP patients differ in their tendency to bleed despite similarly low platelet counts. Moreover, it has been appreciated that, as with the inherited platelet disorders, hemostasis depends not only on platelet count, but also on platelet function.

On the other hand, there are study results addressing the question of whether tests of platelet function may predict bleeding in ITP patients, as most tests of platelet function are affected by low PLTC $[18,19]$. Nowadays, immunofluorescence has many applications in the diagnostic work-up of not only PLTC but also platelet function testing [20, 21]. Platelet analysis by FCM has been applied to the detection of platelet antigens, platelet surface-bound proteins, platelet activation, measurement of reticulated platelets, intracellular calcium studies, and the measurement of platelet microparticles in vivo and in vitro [22-27]. It has been used to evaluate platelet function in research studies, and therefore these assays require clinical validation before they can be used as the standard diagnostic tool in ITP patients.

There were some limitations of our study that should be acknowledged. It was a small group of ITP patients who were enrolled. This was supposed to be an exploratory study in order to gather the information necessary to estimate for a larger follow-up study. We believe that it would be very important to perform an outcome study to prospectively evaluate the PLTC methods, as well as bleeding and thrombosis examination in a further group of patients. Moreover, there should be cost and benefit analyses made before the possible introduction of newer ways of assessing PLTC in ITP patients. 
The second drawback of our study was that we did not investigate the correlation with clinical outcomes, although there were no life-threatening bleeding episodes in any patient in our study.

In our study, similar to previous studies, the immunofluorescent method seems to be the most valuable PLTC method in ITP patients. Clinical applications of FCM analysis have been pursued in individual specialized medical centers so far. This technique has not found widespread adoption in clinical laboratories, mostly due to the difficult standardization process of the method and the inherent biovariability in comparing normal and abnormal platelets.

Despite these hurdles, it seems certain that immunofluorescence in ITP patients will continue to evolve into more practical and robust procedures that may eventually become the standard hematological method, and not only a specialized research tool.

\section{Conclusions}

Accurate PLTC is very important in ITP patients. According to our study, the most reflective test for PLTC is the immunofluorescent method. These findings are especially important during a difficult time in accessing medical care in order to make therapeutic decisions such as a pandemic.

FCM has emerged as an important technology for the study of platelets. Unfortunately, this method is still expensive and labor-intensive, and so should be reserved for selected patients and situations. This also highlights the need to identify novel ways of assessing PLTC in various types of thrombocytopenia.

\section{Authors' contributions}

Michał Witkowski, Piotr Smolewski - study design; Magdalena Witkowska, Michał Witkowski, Agata Majchrzak, Marzena Tybura-Sawicka - data collection; Michał Witkowski, Marzena Tybura-Sawicka - statistical analysis; Michał Witkowski, Piotr Smolewski - data interpretation; Michał Witkowski, Magdalena Witkowska, Tadeusz Robak - manuscript preparation; Magdalena Witkowska, Michał Witkowski - literature search.

\section{Conflicts of interest}

None.

\section{Financial support \\ None.}

\section{Ethics}

The work described in this article has been carried out in accordance with The Code of Ethics of the World Medical Association (Declaration of Helsinki) for experiments involving humans; EU Directive 2010/63/EU for animal experiments; Uniform Requirements for manuscripts submitted to Biomedical journals.

\section{References}

1. Cines DB, Bussel JB, Liebman HA, et al. The ITP syndrome: pathogenic and clinical diversity. Blood. 2009; 113(26): 6511-6521, doi: 10.1182/blood-2009-01-129155, indexed in Pubmed: 19395674.

2. Witkowski M, Witkowska M, Robak T. Autoimmune thrombocytopenia: current treatment options in adults with a focus on novel drugs. Eur J Haematol. 2019; 103(6): 531-541, doi: 10.1111/ejh.13319, indexed in Pubmed: 31449692.

3. Neunert C, Terrell DR, Arnold DM, et al. American Society of Hematology 2019 guidelines for immune thrombocytopenia. Blood Adv. 2019; 3(23): 3829-3866, doi: 10.1182/bloodadvances.2019000966, indexed in Pubmed: 31794604.

4. Liu J, Zheng $X$, Tong $Q$, et al. Overlapping and discrete aspects of the pathology and pathogenesis of the emerging human pathogenic coronaviruses SARS-CoV, MERS-CoV, and 2019-nCoV. J Med Virol. 2020; 92(5): 491-494, doi: 10.1002/jmv.25709, indexed in Pubmed: 32056249

5. Nishioka T, Yamane T, Takubo T, et al. Detection of various platelet-associated immunoglobulins by flow cytometry in idiopathic thrombocytopenic purpura. Cytometry B Clin Cytom. 2005; 68(1): 37-42, doi: 10.1002/cyto.b.20067, indexed in Pubmed: 16184616.

6. Fonio A. Über ein neues Verfahren der Blutplättchenzählung. Dtsch Z Chir. 1912; 117(1-2): 176-194.

7. International Council for Standardization in Haematology Expert Panel on Cytometry International Society of Laboratory Hematology Task Force on Platelet Counting. Platelet Counting by the RBC/Platelet Ratio Method. Am J Clin Pathol. 2001; 115(3): 460-464, doi: 10.1309/ w612-myep-fa7u-8uya.

8. Gatt A, Bonello F, Buttigieg R, et al. Flow cytometry and thromboelastography to assess platelet counts and coagulation in patients with haematological malignancies. Blood Transfus. 2014; 12(4): 479-484, doi: 10.2450/2014.0259-13, indexed in Pubmed: 24960660.

9. Schrezenmeier H, Müller H, Gunsilius E, et al. Anticoagulant-induced pseudothrombocytopenia and pseudoleucocytosis. Thromb Haemost. 1995; 73(3): 506-513, indexed in Pubmed: 7545322.

10. Lippi G, Plebani M. EDTA-dependent pseudothrombocytopenia: further insights and recommendations for prevention of a clinically threatening artifact. Clin Chem Lab Med. 2012; 50(8): 1281-1285, doi: 10.1515/cclm-2012-0081, indexed in Pubmed: 22868791.

11. von dem Borne AE, van der Lelie H, Vos JJ, et al. Antibodies against cryptantigens of platelets. Characterization and significance for the serologist. Curr Stud Hematol Blood Transfus. 1986; 52: 33-46, indexed in Pubmed: 2421983.

12. Bowles KM, Bloxham DM, Perry DJ, et al. Discrepancy between impedance and immunofluorescence platelet counting has implications for clinical decision making in patients with idiopathic thrombocytopenia purpura. Br J Haematol. 2006; 134(3): 320-322, doi: 10.1111/j.1365-2141.2006.06165.x, indexed in Pubmed: 16848774.

13. Harrison P, Horton A, Grant D, et al. Immunoplatelet counting: a proposed new reference procedure. Br J Haematol. 2000; 108(2): 228-235, doi: 10.1046/j.1365-2141.2000.01846.x, indexed in Pubmed: 10691847.

14. Hoffmann JJ. Reticulated platelets: analytical aspects and clinical utility. Clin Chem Lab Med. 2014; 52(8): 1107-1117, doi: 10.1515/ cclm-2014-0165, indexed in Pubmed: 24807169. 
15. Pedersen $\mathrm{OH}$, Larsen $\mathrm{ML}$, Grove $\mathrm{EL}$, et al. Platelet characteristics in patients with essential thrombocytosis. Cytometry B Clin Cytom. 2018; 94(6): 918-927, doi: 10.1002/cyto.b.21642, indexed in Pubmed: 29790256.

16. Tanaka $Y$, Tanaka $Y$, Gondo $K$, et al. Performance evaluation of platelet counting by novel fluorescent dye staining in the XN-series automated hematology analyzers. J Clin Lab Anal. 2014; 28(5): 341-348, doi: 10.1002/jcla.21691, indexed in Pubmed: 24648166.

17. Sun $\mathrm{Yi}, \mathrm{Hu} Z$, Huang $\mathrm{Z}$, et al. Compare the accuracy and precision of Coulter LH780, Mindray BC-6000 Plus, and Sysmex XN-9000 with the international reference flow cytometric method in platelet counting. PLoS One. 2019; 14(5): e0217298, doi: 10.1371/journal. pone.0217298, indexed in Pubmed: 31125378.

18. Dickerhof $R$, von Ruecker A. Enumeration of platelets by multiparameter flow cytometry using platelet-specific antibodiesand fluorescent reference particles. Clinical \& Laboratory Haematology. 1995; 17(2): 163-172, indexed in Pubmed: 536420.

19. Panzer S, Rieger M, Vormittag R, et al. Platelet function to estimate the bleeding risk in autoimmune thrombocytopenia. Eur J Clin Invest. 2007; 37(10): 814-819, doi: 10.1111/j.1365-2362.2007.01855.x, indexed in Pubmed: 17727674.

20. van Bladel ER, Laarhoven AG, van der Heijden LB, et al. Functional platelet defects in children with severe chronic ITP as tested with 2 novel assays applicable for low platelet counts. Blood. 2014; 123(10): 1556-1563, doi: 10.1182/blood-2013-08-519686, indexed in Pubmed: 24385537.
21. van Asten I, Schutgens REG, Baaij M, et al. Validation of flow cytometric analysis of platelet function in patients with a suspected platelet function defect. J Thromb Haemost. 2018; 16(4): 689-698, doi: 10.1111/jth.13952, indexed in Pubmed: 29337406.

22. Israels SJ. Laboratory testing for platelet function disorders. Int J Lab Hematol. 2015; 37(Suppl 1): 18-24, doi: 10.1111/ijlh.12346, indexed in Pubmed: 25976956.

23. Smith GA, Ranasinghe E, Ouwehand WH. The importance of using multiple techniques for detection of platelet antibodies. Vox Sang. 2007; 93(4): 306-308, doi: 10.1111/j.1423-0410.2007.00969.x, indexed in Pubmed: 18070274.

24. Rosenfeld CS, Nichols G, Bodensteiner DC. Flow cytometric measurement of antiplatelet antibodies. Am J Clin Pathol. 1987; 87(4): 518-522, doi: 10.1093/ajcp/87.4.518, indexed in Pubmed: 3103420.

25. Romero-Guzman LT, Lopez-Karpovitch X, Paredes R, et al. Detection of platelet-associated immunoglobulins by flow cytometry for the diagnosis of immune thrombocytopenia: a prospective study and critical review. Haematologica. 2000; 85(6): 627-631, indexed in Pubmed: 10878748.

26. Tomer A. Autoimmune thrombocytopenia: determination of platelet-specific autoantibodies by flow cytometry. Pediatr Blood Cancer. 2006; 47(5 Suppl): 697-700, doi: 10.1002/pbc.20987, indexed in Pubmed: 16933272.

27. Hézard N, Simon G, Macé C, et al. Is flow cytometry accurate enough to screen platelet autoantibodies? Transfusion. 2008; 48(3): 513-518, doi: 10.1111/j.1537-2995.2007.01556.x, indexed in Pubmed: 18067500. 